\title{
Registres pour la recherche sur les soins et l'assurance-qualité
}

Nicole Steck ${ }^{a}$, Stefanie Hostettler ${ }^{\mathrm{b}}$, Esther Kraft ${ }^{\mathrm{b}}$, Claudia Berlin ${ }^{\mathrm{a}}$, Adrian Spörria, Marcel Zwahlen ${ }^{\mathrm{a}}$

${ }^{a}$ Institut de médecine sociale et préventive de I'Université de Berne; ${ }^{b} \mathrm{FMH}$, division Données, démographie et qualité

Le nombre de registres médicaux augmente en Suisse. Spécifiques à un traitement ou à une maladie, ces registres répondent à un choix délibéré ou une obligation et poursuivent des objectifs différents. Ils constituent un pilier important de l'assurance-qualité et de la recherche (sur les soins) mais ne permettent pas de résoudre toutes les questions pressantes. Les informations qu'ils contiennent servent de plus en plus aux évaluations des technologies de la santé (HTA). L'utilité des registres dépend de leur qualité, mesurée à l'aune de la couverture et de la représentativité des données qu'ils recensent mais aussi de leur exhaustivité et de leur exactitude, notamment si les informations relatives à l'évolution de la maladie après le diagnostic et le premier traitement sont saisies de manière fiable.

Près d'une centaine de registres médicaux sont actuellement répertoriés sur la Plateforme suisse des registres médicaux de la FMH (www.fmh.ch/fr/services/ qualite/plateforme_registres-medicaux.html) [1]. Leur nombre augmente à chaque nouvelle actualisation et couvre un large éventail de thèmes qui vont de $\mathrm{A}$ comme AMDS pour Absolute Minimal Data Set sur les

\section{Plateforme suisse des registres} médicaux

L'Académie suisse pour la qualité en médecine (ASQM) recense les registres médicaux depuis 2012 sur son site internet [1]. Grâce à cette plateforme en ligne, elle montre le large éventail de ce domaine et offre la possibilité de trouver en quelques clics le registre recherché.

Cette banque de données a pour vocation de mettre les informations récoltées sur les registres à la disposition des personnes intéressées, d'améliorer la collaboration entre les responsables de registres et d'aider les personnes souhaitant créer un registre. Outre une brève description des registres, on y trouve des renseignements sur la région géographique, le type de données, la méthode, la population, les possibilités de participation, l'accès aux données, le financement ou encore l'établissement de rapports. Régulièrement mise à jour par I'ASOM, la plateforme en ligne lui sert de base pour ses prochains travaux dans le domaine des registres.

www.fmh.ch $\rightarrow$ Qualité $\rightarrow$ Plateforme suisse des registres médicaux activités dans le domaine de l'anesthésiologie à Z pour ZDR (en allemand), le registre dosimétrique de l'OFSP, un registre central des doses accumulées par les personnes exposées aux rayonnements dans l'exercice de leur profession en Suisse. Mais un registre médical, qu'est-ce que c'est exactement, à quoi sert-il et quelles exigences doit-il remplir? La diversité des définitions trouvées dans la littérature scientifique est à l'image des différences qui existent entre les registres. De manière consensuelle, on parle d'un "recueil systématique de données de même nature sur un collectif de recherche déterminé» $[2,3]$. Un document européen de 2015 poursuit [4]: «Les registres constituent un système organisé pour recueillir, analyser et diffuser des données et des informations d'un groupe de personnes défini par une maladie déterminée, un état, une exposition ou une prestation médicale; et ils servent un objectif scientifique, clinique et/ou politique fixé préalablement."

Les diverses exigences auxquelles doivent répondre les registres expliquent aussi les différences parfois importantes et les degrés de détail différents de leurs définitions. Certains registres, notamment dans le domaine des maladies infectieuses, servent aussi bien de système de détection précoce que d'évaluation à long terme des mesures de contrôle, alors que d'autres accompagnent la recherche ou servent à établir la trans- 
parence. La Suisse exige par exemple de signaler plus de 50 maladies différentes à l'Office fédéral de la santé publique, dont le VIH, la malaria, la rougeole, la tuberculose et, à partir de 2020, les cancers. Majoritairement facultative, la participation aux registres est souvent

\section{La Suisse exige par exemple de signaler plus de 50 maladies différentes à l'Office fédéral de la santé publique.}

motivée par des médecins spécialistes ou des sociétés de discipline médicale, mais aussi dans certains cas par des groupes de patients. Afin de mieux distinguer les différentes formes de registres, la littérature propose de les catégoriser par exemple en registres de santé publique, registres médicaux, registres épidémiologiques, registres cliniques et registres de patients [2, 3 , 5, 6] (tab. 1). Notons cependant que cette distinction, comme celle en partie aussi des cohortes, n'est pas toujours évidente et qu'un registre peut poursuivre plusieurs objectifs différents.

\section{Collecter des données, oui mais correctement}

Indépendamment de cet objectif, un registre doit satisfaire à certains critères qualité; c'est le seul moyen de justifier le temps, l'argent et l'organisation largement

Tableau 1: Catégorisation possible des différents types de registres.

\begin{tabular}{|c|c|c|c|c|}
\hline Genre de registres & Critères de sélection & Résultats & Quelles comparaisons? & $\begin{array}{l}\text { Exemples de conclusions } \\
\text { possibles }\end{array}$ \\
\hline $\begin{array}{l}\text { Registres de santé publique } \\
\text { Exemples: } \\
\text { Registre des personnes après } \\
\text { administration de vaccins } \\
\text { Registre des personnes dépistées }\end{array}$ & $\begin{array}{l}\text { Région/participation } \\
\text { à un programme de } \\
\text { vaccination ou de } \\
\text { dépistage }\end{array}$ & $\begin{array}{l}\text { Couverture du programme } \\
\text { dans la population cible } \\
\text { Effets secondaires après } \\
\text { les vaccinations }\end{array}$ & $\begin{array}{l}\text { Comparaison de la fréquence } \\
\text { des effets secondaires en } \\
\text { fonction du type de vaccin } \\
\text { Comparaison des investigations } \\
\text { consécutives à un dépistage du } \\
\text { cancer du sein par région }\end{array}$ & $\begin{array}{l}\text { Couverture du programme } \\
\text { plus faible chez les jeunes } \\
\text { que chez les adultes } \\
\text { L'incidence des effets se- } \\
\text { condaires après un vaccin } \\
\text { trivalent ne diffère pas de } \\
\text { l'incidence après un vaccin } \\
\text { bivalent }\end{array}$ \\
\hline $\begin{array}{l}\text { Registres médicaux } \\
\text { Exemples: } \\
\text { Registre des patients avec } \\
\text { une prothèse (opération) } \\
\text { Registre des patients ayant subi } \\
\text { une intervention cardiaque }\end{array}$ & $\begin{array}{l}\text { Maladie ou interven- } \\
\text { tion médicale/traite- } \\
\text { ment déterminé }\end{array}$ & Evolution, complication & $\begin{array}{l}\text { Comparaison du traitement } A \\
\text { et du traitement } B \text { ou avec les } \\
\text { patients sans traitement } \\
\text { Comparaison de différents } \\
\text { hôpitaux }\end{array}$ & $\begin{array}{l}\text { Le besoin de correction est } \\
\text { plus important avec l'im- } \\
\text { plant A qu'avec l'implant B } \\
\text { La mortalité après une chirur- } \\
\text { gie cardiaque est plus élevée } \\
\text { dans une clinique que dans les } \\
\text { autres cliniques pour les } \\
\text { facteurs de risque comme } \\
\text { l'Euroscore II }\end{array}$ \\
\hline $\begin{array}{l}\text { Registres épidémiologiques } \\
\text { Exemples: } \\
\text { Registre de tous les cas de décès }\end{array}$ & $\begin{array}{l}\text { Moment du } \\
\text { diagnostic/région }\end{array}$ & Incidence, mortalité & $\begin{array}{l}\text { Comparaison avec d'autres } \\
\text { moments du diagnostic } \\
\text { Comparaison régionale }\end{array}$ & $\begin{array}{l}\text { Recul des infarctus du myo- } \\
\text { carde ayant entraîné la mort } \\
\text { par rapport à l'année précé- } \\
\text { dente }\end{array}$ \\
\hline
\end{tabular}

avec la cause

L'incidence du cancer du sein standardisée selon l'âge chez les femmes de moins de 40 ans augmente

Registre des personnes avec

Registres cliniques

Centre hospitalie traitant

Exemples:

Patientes d'un hôpital avec le

diagnostic d'un cancer du sein

Registre des bénéficiaires

d'une transplantation

\section{Registre des patients}

(partiellement aussi les cohortes)

Maladie déterminée

Evolution, traitements suivis, qualité de vie

Exemples:

Registre des personnes atteintes

de sclérose en plaques

Registre des personnes atteintes

d'une maladie neuromusculaire

\section{Evolution après traitement, Données sur l'évolution chez des - survie à 5 ans selon changement ou arrêt du groupes de patients définis, traitement moment du diagnostic est la même qu'il y a 10 ans}

La fréquence du changement de traitement est plus élevée à un stade plus avancé de la maladie

Comparaison de l'évolution après différents traitements, éventuellement différentes variantes génétiques de la maladie
La proportion de personnes atteintes de sclérose en plaque exerçant une activité professionnelle augmente

Une partie des paralysies chez les personnes atteintes de lésions de la moelle épinière est réversible 
investis pour le mettre en place et le gérer [7, 8]. Un registre tenu sans la diligence requise est, dans le pire des cas, non seulement inutile mais il peut aussi conduire à des informations non fiables et donc à des conclusions ou des décisions erronées.

La continuité constitue un autre point important pour un registre. Il doit être conçu de telle sorte que son utilisation au quotidien reste réaliste, même une fois l'euphorie du début retombée. Son financement devrait aussi être garanti à long terme, tout comme la participation des organismes qui livrent les informations.

Le registre doit être conçu de telle sorte que son utilisation au quotidien reste réaliste, même une fois l'euphorie du début retombée.

Un autre critère primordial pour la qualité d'un registre est son exhaustivité, et ce sur plusieurs plans: d'un côté, tous les cas visés (correspondant aux exigences) doivent, si possible, être enregistrés, ou avoir la possibilité de l'être. Cela signifie que dans une clinique qui répertorie toutes les infections, on ne peut pas avoir un service qui recense chaque cas méticuleusement tandis que dans un autre service, les infections sont seulement enregistrées lorsque quelqu'un a le temps de le faire. L'obligation d'inscrire les cas ne veut pas non plus dire que le registre sera exhaustif. On estime par exemple que le nombre de cas de chlamydiose, qu'il est obligatoire d'annoncer, ne représente qu'une part infime de cette maladie souvent asymptomatique. De l'autre côté, toutes les informations enregistrées par cas doivent avoir le même degré de détail. Si une clinique signale par exemple tous les cas mais

\section{Toutes les informations enregistrées par cas doivent avoir le même degré de détail.}

pas toutes les complications dans un registre, on risque d'en tirer de mauvaises conclusions. C'est la même chose si les complications enregistrées se limitent à celles consécutives à une opération, sans reprendre les complications sur les patients n'ayant finalement pas subi d'intervention.

La cohérence des données est un autre point crucial et exigeant. Mettre en place des directives claires, reposant dans la mesure du possible sur des standards nationaux ou internationaux, permet de garantir l'uniformité des enregistrements, indépendamment des personnes qui annoncent les cas. C'est particulièrement important lorsque l'information collectée laisse place à l'interprétation.

Pour qu'un registre fonctionne, il est essentiel que les saisies soient actuelles. Si elles sont livrées avec plu-
Groupe de dialogue: recherche

\section{prioritaire sur les soins}

Pour le corps médical, la recherche sur les soins constitue un domaine scientifique important et porteur d'avenir. Dans un secteur de la santé en pleine mutation (nouveaux modèles de financement, évolutions démographiques, hausse des coûts, etc.), il est impératif que la recherche sur les soins bénéficie d'un ancrage académique. Afin de pouvoir créer les bases scientifiques d'une recherche indépendante dénuée de tout intérêt particulier, la Fédération des médecins suisses (FMH) et Newlndex soutiennent ensemble la recherche prioritaire sur les soins de l'Institut de médecine sociale et préventive de I'Université de Berne. A l'instar d'un forum, un groupe de dialogue a pour but de solliciter l'échange d'informations: les représentants des organisations et des groupes de recherche discutent régulièrement des travaux en cours et des projets à venir dans le domaine de la recherche sur les soins. Par ailleurs, le groupe de dialogue vise à sensibiliser le corps médical à ce domaine scientifique et à en favoriser l'acceptation. Il est ouvert aux propositions en ce qui concerne les sujets à traiter, les questions, les discussions ou les demandes d'informations supplémentaires. La division Données, démographie et qualité (DDQ) de la FMH assure la coordination du groupe de dialogue et se tient à disposition pour tout complément d'information par courriel à ddq[at]fmh.ch ou par téléphone au 0313591111.

sieurs mois de retard, toute demande de renseignement devient difficile, et ce au détriment des évaluations ultérieures et de la vue d'ensemble des données. Un autre aspect primordial est aussi la pertinence des informations collectées. Il peut être tentant de commencer par recueillir toutes les données possibles. Mais au moment de structurer un registre, il est très important de fixer clairement les objectifs poursuivis et de définir en conséquence les données à collecter.

La protection de la personnalité doit par ailleurs être garantie. Comment les données sont-elles anonymisées, qui a accès à la clé d'anonymisation, quelles données sont transmises à qui et que se passe-t-il lorsqu'un patient retire son consentement?

La validation permet de garantir la qualité des données. Les informations collectées doivent être systématiquement contrôlées pour détecter les erreurs et en vérifier la plausibilité afin de maintenir un niveau élevé de la qualité.

En 2016, la FMH a publié des «Recommandations concernant la création et la gestion de registres dans le domaine de la santé» conjointement avec l'ANQ, H+, l'ASSM et Médecine universitaire Suisse [8]. Ces recommandations communes ont débouché sur une liste de contrôle permettant de vérifier un registre en 7 étapes (1. Planification d'un registre: clarifications préalables; 2. Conception du registre; 3. Compétences relatives à la gestion du registre; 4 . Protection et souveraineté des 
données; 5. Collecte et utilisation des données; 6. Assurance-qualité; 7. Changement d'objectif et suppression du registre).

\section{L'utilité des données collectées}

Même si un registre est géré de manière optimale avec des données exhaustives, actuelles et pertinentes, il ne présentera pas beaucoup d'intérêt si les données qu'il contient ne sont pas analysées ni utilisées.

La manière d'analyser et d'utiliser les données dépend entièrement de la finalité du registre. Les registres obligatoires de la Confédération servent avant tout de système de dépistage précoce et de surveillance. On peut lire sur le site de l'OFSP: «Dans l'optique de prévenir et d'éviter les dommages, les interventions dans la dynamique d'un événement épidémiologique nécessitent le plus souvent de pouvoir dépister et déclarer les menaces pesant sur la santé publique dans les plus brefs délais.» Les registres épidémiologiques représentatifs d'un domaine ou d'un groupe de patients permettent aussi d'identifier les facteurs de risque possibles. Les

\section{Lorsque leur utilisation est optimale, les registres médicaux peuvent permettre de comprendre certaines situations ou de soulever des questions pour la recherche.}

registres médicaux sur une maladie ou un traitement médical spécifiques constituent souvent des ensembles de données extraordinaires, indispensables à l'évolution de la prise en charge médicale.

Comme les données des différents registres couvrent des domaines respectifs circonscrits, il est souvent intéressant pour la recherche de les combiner entre eux ou d'associer un registre à des informations officielles. Mais les possibilités restent très limitées soit en raison d'obstacles techniques ou de la réglementation sur la protection des données.

Lorsque leur utilisation est optimale, les registres médicaux peuvent permettre de comprendre certaines situations ou de soulever des questions pour la recherche mais ils ne remplacent généralement pas les études randomisées. Pour la plupart, ils ne permettent pas en effet de distinguer entre les causes et les effets. De plus, les données enregistrées ne sont souvent pas représentatives car elles correspondent à un choix défini et déterminé notamment par la participation des cliniques, les différentes motivations des patients ou les différences géographiques.

Les registres peuvent aussi jouer un rôle primordial lors d'études randomisées. Pour des maladies isolées, notamment dans le domaine des maladies orphelines, il est souvent difficile de réunir suffisamment de sujets susceptibles de participer à une étude. Les registres peuvent donc offrir des contacts utiles. En revanche, lorsqu'ils sont facultatifs, il faut tenir compte du fait que les patients recensés ne sont pas nécessairement représentatifs car ce seront plutôt ceux plus touchés ou ceux d'un médecin plus particulièrement motivé par tel ou tel registre qui seront enregistrés.

\section{L'assurance-qualité dans la prise en charge médicale}

Dans le domaine de l'assurance-qualité des dispositifs médicaux implantés, un rôle important revient aux registres, qu'ils soient facultatifs ou organisés par l'Etat, car ils ont notamment permis de conclure que les prothèses de hanche métal-métal n'étaient pas suffisamment sûres [9-11]. En revanche, aucune vue d'ensemble claire ne permet d'identifier les pays dans lesquels il existe des registres de dispositifs médicaux implantables [12]. Une telle vue d'ensemble ne verra le jour qu'avec le renforcement de la mission de surveillance des autorités acté par l'adaptation de la réglementation européenne pour les dispositifs médicaux (https:// www.swissmedic.ch/swissmedic/fr/home/dispositifsmedicaux/nouveaux-reglements-europeens-rdm-rdiv. html). En traumatologie, les informations d'un registre international devraient améliorer les traitements dans les services des urgences [13]. En Suisse, il est prévu de garantir la qualité des traitements transplantatoires en institutionnalisant la cohorte de transplantation [14]. Là aussi, on a pu voir qu'il n'était pas toujours simple de distinguer entre registre et étude de cohorte. Aux Etats-Unis par exemple, la saisie systématique des transplantions est considérée comme un registre [15]. Un objectif clairement déclaré est de recenser l'évolution de la santé et les autres traitements dispensés aux personnes transplantées tout au long de leur vie après

\section{Recommandations communes pour les registres de santé}

Conjointement avec I'ANQ, H+, I'ASSM et unimedsuisse, I'ASOM a élaboré des recommandations concernant la création et la gestion de registres dans le domaine de la santé. Visant à garantir la qualité, ces recommandations communes contiennent des standards minimaux, notamment en matière de protection et de qualité des données [8]. Suite à un projet pilote au cours duquel dix registres médicaux ont été examinés à la lumière de ces recommandations. Les registres vérifiés font l'objet d'une évaluation quant à leur composition et aux éventuelles possibilités d'amélioration. Dans le même temps, les recommandations seront étendues afin de continuer à soutenir la qualité des registres suisses.

www.asqm.ch $\rightarrow$ Publications $\rightarrow$ Recommandations registres 
la transplantation. Cela exige d'une part des visites régulières en clinique mais aussi l'accès réglementé aux données du registre de la population ou du registre des décès.

Inversement, le Registre suisse de la sclérose en plaques (SEP) est un exemple de ce qu'on appelle un re-

\section{Il n'est pas toujours simple de distinguer entre registre et étude de cohorte.}

gistre bottom-up [16]. Mis en place et financé par la Société suisse de la sclérose en plaques, ce registre vise à documenter la qualité de vie des personnes atteintes de SEP en Suisse, en plus d'examiner les questions épidémiologiques.

Dans le cadre d'une standardisation des HTA au niveau européen, une enquête a été récemment menée pour savoir sous quelle forme et dans quelles conditions les informations des registres étaient consultées par les différentes autorités en charge des HTA [17]. Elle a fait ressortir que les données des registres étaient utilisées plus souvent qu'on ne le pensait, mais aussi que les registres devaient répondre à plusieurs catégories de critères qualité pour être utilisés dans les HTA. Il est maintenant prévu de développer un instrument d'évaluation de la qualité sur la base de cette enquête.

\section{Perspectives}

Les registres peuvent être considérés comme une ramification du big data et, à ce titre, ils gagneront probablement encore en importance ces prochaines années. Loin d'être une arme magique, ils exigent au contraire une structure bien pensée et beaucoup de diligence afin de pouvoir contribuer à la recherche et à l'assu-

\section{Il serait judicieux d'utiliser un seul et même numéro d'identification (anonyme) pour les différents registres et les données de routine.}

rance-qualité. Ce qui s'applique de manière générale au big data vaut également pour les registres: on ne fait pas une bonne tarte avec des pommes pourries. La possibilité de liaison et l'harmonisation entre les données des registres et celles de routine sont primordiales pour le développement et l'utilisation à venir des registres de santé. Depuis 2017, le réseau Swiss Personalized Health Network (SPHN) pose «les bases nécessaires pour faciliter les projets de recherche tel qu'un système national d'échange des données de santé» (https://www.sphn.ch). Par ailleurs, il serait judicieux d'utiliser un seul et même numéro d'identification (anonyme) pour les différents registres et les données de routine mais le volet juridique et la protection des données doivent encore être clarifiés. Il faut espérer que le Programme national de recherche «Système de santé» (PNR 74, http://www.nfp74.ch) et d'autres programmes pour renforcer la recherche sur les soins offriront suffisamment d'occasions de progresser dans ce domaine.

\section{Références}

1 Hostettler S, Hersperger M. Clôture du projet «Plateforme suisse des registres médicaux»: Tour d'horizon des registres médicaux de Suisse. Bull Med Suisses. 2012;93:99-2.

2 Weddell JM. Registers and registries: a review. Int J Epidemiol. 1973;2(3):221-8.

3 Alter M. Medical registers. Advances in neurology. 1978;19:121-39.

4 Zaletel M, Kralj M. Methodological guidelines and recommendations for efficient and rational governance of patient registries. Ljubljana: National Institute of Public Health; 2015.

5 Mathis-Edenhofer S, Piso B [Types of medical registries - definitions, methodological aspects and quality of the scientific work with registries]. Wien Med Wochenschr. 2011;161(23-24):580-90.

6 Mathis S, Wild C. Register für klinische und gesundheitsökonomische Fragestellungen: Einsatzbereiche von kardiovaskulären, wirbelsäulenspezifischen und neurologischen Registern und Good Practice Strategien für die Arbeit mit Registern. HTA Projektbericht \#11, 2008.

7 Hostettler S, Hersperger M, Herren D. Registres médicaux: où se trouve la clé du succès? Bull Med Suisses. 2012;93:1251-5.

$8 \mathrm{ANQ}, \mathrm{FMH}, \mathrm{H}+$, et al. Recommandations concernant la création et la gestion de registres dans le domaine de la santé. Berne; 2016

9 Smith AJ, Dieppe P, Howard PW, et al. Failure rates of metal-on-metal hip resurfacings: analysis of data from the National Joint Registry for England and Wales. The Lancet. 2012;380(9855):1759-66.

10 Sedrakyan A. Metal-on-metal failures - in science, regulation, and policy. The Lancet. 2012;379(9822):1174-6.

11 Sedrakyan A, Paxton E, Graves S, et al. National and international postmarket research and surveillance implementation: achievements of the International Consortium of Orthopaedic Registries initiative. The Journal of bone and joint surgery American volume. 2014;96(Suppl 1):1-6.

12 Niederlander C, Wahlster P, Kriza C, et al. Registries of implantable medical devices in Europe. Health Policy. 2013;113(1-2):20-37.

13 Hartel MJ, Jordi N, Evangelopoulos DS, et al. Optimising care in a Swiss University Emergency Department by implementing a multicentre trauma register (TARN): report on evaluation, costs and benefits of trauma registries. Emerg Med J. 2011;28(3):221-4.

14 Koller MT, van Delden C, Müller NJ, et al. Design and methodology of the Swiss Transplant Cohort Study (STCS): a comprehensive prospective nationwide long-term follow-up cohort. European Journal of Epidemiology. 2013;28(4):347-55.

15 Organ Procurement and Transplantation Network and Scientific Registry of Transplant Recipients 2010 Data Report. American Journal of Transplantation. 2012;12(s1):1-156.

16 Steinemann N, Kuhle J, Calabrese P, et al. The Swiss Multiple Sclerosis Registry (SMSR): study protocol of a participatory, nationwide registry to promote epidemiological and patient-centered MS research. BMC Neurology. 2018;18(1):111.

17 Mandeville KL, Valentic M, Ivankovic D, et al. Quality assurance of registries for health technology assessment. International Journal of Technology Assessment in Health Carem 2018;34(4):360-7. 